\title{
Análisis legal y doctrinario del Ordenamiento y Planificación Territorial en Chile, características, relación y diferencias.
}

\author{
Mindy Fuentes Jara \\ Facultad de Ingeniería Geográfica, Universidad de Santiago, Enrique Kirberg Baltiansky \# 03, \\ Estación Central, Santiago - CHILE. E-mail: mindy.fuentes@usach.cl \\ Dirección postal: Pasaje Cillero 29, Rancagua, Chile.
}

\section{Resumen}

En este trabajo se analiza desde el punto de vista legal y doctrinario dos conceptos que se han confundido en el desarrollo de la gestión del territorio, que son el Ordenamiento y la Planificación Territorial. El objetivo del análisis es delimitar sus alcances y funciones, determinando sus características y diferencias, además de la relación entre uno y otro concepto. La importancia del presente estudio, es el análisis crítico de estos conceptos, a la luz de las falencias que existen en el ordenamiento jurídico chileno para definirlos y determinar la relación entre ellos, lo cual ha sido suplido por conceptos doctrinarios que logran explicar cuál es su relación, características, diferencias y funciones en el desarrollo territorial.

Palabras clave: Ordenamiento Territorial, Planificación Territorial, Plan Regional de Ordenamiento Territorial.

\begin{abstract}
In this paper we analyzed from the legal and doctrinal point of view of two concepts that have been confused in the development of land management, which are the Planning and Territorial Planning. The objective of the analysis is to define its scope and functions, determining their characteristics and differences, besides the relationship between two concepts. The importance of this study is the critical analysis of these concepts, in light of the shortcomings that exist in the Chilean legal system to define and determine the relationship between them, which has been supplemented
\end{abstract}


Sustainability, Agri, Food and Environmental Research 3(2), 2015: 12-21

ISSN: 0719-3726

by doctrinal concepts that manage to explain their relationship, features, and functions differences in territorial development.

Key Word: Zoning, Planning, Regional Land Use Plan.

Concepto de Ordenamiento Territorial en Chile.

En nuestro país el Ordenamiento Territorial no ha sido definido por el ordenamiento jurídico. Sin embargo, el concepto de Ordenamiento Territorial, ha sido discutido por diferentes autores, donde encontramos desde el análisis conceptual hasta la crítica que se traza en relación a la confusión del concepto con la Planificación Territorial. Bustos, por ejemplo, señala que en Chile hay distancia de una concepción precisa de Ordenamiento Territorial, tendiendo siempre hacia una "asociación directa que se hace del Ordenamiento Territorial con el desarrollo de los Instrumentos chilenos de Planificación Territorial"1, ejercicio que de acuerdo a lo que el autor señala es desacertado, ya que la Planificación Territorial es el proceso de planeación de un territorio a escala local, a través de los Instrumentos de Planificación Territorial.

Bustos hace referencia al documento "Ordenamiento Territorial en Chile (1993) del ministerio encargado de las políticas habitacionales y del desarrollo urbano.", el cual concluye que en Chile, la normativa se ha preocupado de regular planificación urbana sin concebir un concepto de Ordenamiento Territorial. Así mismo indica, que el Ordenamiento Territorial está encargado de definir los principios que se establecen como país para ocupar el territorio, lo que se diferencia de la planificación, que sería la ejecución de estos principios².

Según Romero y Vásquez ${ }^{3}$, el territorio es un espacio complejo donde convergen el medio natural y la sociedad, por ello requiere de un tratamiento especial por parte del Estado, donde se le reconozca recogiendo el principio de bien común, estableciendo políticas públicas y sistemas jurídicos que protejan y regulen hacia la administración ecuánime de éste. La crítica que desarrollan los autores, destaca que la falta de una conceptualización y regulación del Ordenamiento Territorial en Chile se ha provocado a causa de los intereses del mercado, destacando que el territorio es un

\footnotetext{
${ }^{1}$ BUSTOS Arancibia, Nelson, "El ordenamiento y planificación territorial en Chile: elementos para la discusión", Revista de Geografía Norte Grande, Santiago, 1998, 25: 49-53, pág. 1

${ }^{2}$ BUSTOS, Op. cit. pág. 50

${ }^{3}$ ROMERO Hugo, VASQUEZ Alexis, "Pertinencia y Significado del Ordenamiento Territorial en Chile", Urbano, Red de Revistas científicas de América Latina, el Caribe, España y Portugal, Sistema de Información Científica, vol. 8, 11 julio 2005, pp.91-99, Universidad del Bio Bio, Chile.[consulta en línea abril 2014noviembre 2014] < http://www.redalyc.org/articulo.oa?id=19801112 >
} 
Sustainability, Agri, Food and Environmental Research 3(2), 2015: 12-21 ISSN: 0719-3726

espacio que debe ser observado en pos del bien común de toda la sociedad. Señalan que esta problemática podría solucionarse en la práctica con una regulación idónea que propenda al desarrollo sustentable, fundamentando que "el territorio y muchos de sus componentes forman parte del bien común y por ello, la única manera de protegerlos y asegurar su permanencia es mediante regulaciones y normas dictadas y fiscalizadas por el estado en representación de la totalidad de la sociedad."4

Del análisis anterior, tanto de Bustos como de Romero y Vásquez, podemos concluir que el Ordenamiento Territorial debiera ser concebido como una política que establezca las directrices y principios de la ocupación del territorio, y que dentro de sus lineamientos debe incorporarse el principio de desarrollo sustentable y el respeto por el bien común, a través de una regulación general que sea la base de la Planificación Territorial (ejecución y técnica) la cual es la aplicación de las directrices establecidas por la política de Ordenamiento Territorial, en los niveles que la ley determina, ya sea nacional, regional, intercomunal, o local.

Del mismo modo, el Ordenamiento Territorial debe resolver las "disparidades espaciales", pues uno de sus principales objetivos es ser una política pública nacional destinada a fortalecer el desarrollo equitativo en el territorio, en su aspecto económico, social, cultural, patrimonial, industrial y medio ambiental. Es de esta opinión Federico Arenas $^{5}$, que compara el caso de Chile con otros países de América Latina, relativa a la existencia de una política de Ordenamiento Territorial que se ocupe de las disparidades espaciales. Concluye que ni América Latina ni Chile poseen una política que pueda enfrentar la disparidad de desarrollo en el territorio a través del Ordenamiento Territorial. A su vez indica que Boisier ${ }^{6}$ elabora una matriz completa de política pública de Ordenamiento Territorial, en base a cuatro ejes: 1. La Planificación Territorial a través de los distintos IPTs que existen; 2. La descentralización; 3. Fomento al crecimiento económico territorialmente diferenciado; e 4. Impulso al desarrollo local.

Arenas $^{7}$ concluye que de acuerdo al modelo de Boisier, en Chile no hay Ordenamiento Territorial, dado a que los diferentes ejes que este debe contener (según el modelo de Boisier) no están contenidos en las políticas públicas sobre la materia. Sin embargo sí reconoce un interés de nuestro país por desarrollar políticas sectoriales en cuanto a la administración del territorio, sin

\footnotetext{
${ }^{4}$ Op. cit. pág. 99

${ }^{5}$ ARENAS, Federico, Seminario Regional, Ordenamiento Territorial de la Región de Tarapacá, sus alcances y desafíos, Iquique, 12 de abril 2011

${ }^{6}$ BOISIER, Sergio, en ARENAS, Federico, Seminario Regional, Ordenamiento Territorial de la Región de Tarapacá, sus alcances y desafíos, Iquique, 12 de abril 2011.

${ }^{7}$ ARENAS, Federico, Seminario Regional, Ordenamiento Territorial de la Región de Tarapacá, sus alcances y desafíos, Iquique, 12 de abril 2011
} 
Sustainability, Agri, Food and Environmental Research 3(2), 2015: 12-21 ISSN: 0719-3726

embargo evidencia la inexistencia de una única institucionalidad encargada del desarrollo del Ordenamiento Territorial, por el contrario, existen diferentes instituciones encargadas de la ejecución de la planificación urbana en sus distintos niveles de acción, nacional, regional, intercomunal y comunal. Cada una de ellas con sus correspondientes responsabilidades sectoriales en materia de infraestructura urbana, rural, instrumentos normativos de carácter urbano, o instrumentos de carácter indicativo.

En síntesis, Arenas describe, que en el caso de Chile, no existe una política de Ordenamiento Territorial, y si bien existe un esfuerzo de instaurarla, ha sido parcial, destinado a dar enfoques urbanísticos, o en su caso, ambientales, en algunos casos débiles, sin confluir en una política pública específica que establezca directrices idóneas.

Además, como sostiene Correa, Chile ha adoptado un “...modelo, erguido predominantemente sobre normativa urbano-regional, que además se ve complementada por un gran número de normas de carácter sectorial ...”, además teniendo presente que si se le compara con sistemas de otros países latinoamericanos, encontramos una "ausencia de políticas nacionales que orienten las acciones del Ordenamiento Territorial en los distintos ámbitos territoriales de actuación, así como una cobertura territorial limitada de los planes, debilidad y desorden normativo y ausencia de una organización institucional adecuada y coordinada para la gestión del ordenamiento ..."8.

También es de la opinión de la falta de una política pública de Ordenamiento Territorial en Chile, Eduardo Cordero, el que en su artículo "El Derecho urbanístico, los Instrumentos de Planificación Territorial y el régimen jurídico de los bienes públicos", señala que Chile se ha enfocado a la Planificación Territorial a través de los Instrumentos de Planificación Territorial, previstos en la legislación urbanística, indicando además que no hemos sido capaces de realizar una Planificación Territorial adecuada, descentralizada, sino más bien, tendiente a plasmar un "sistema de Planificación Territorial de una enorme centralización"9 y los de nivel supralocal son insuficientes, según su conclusión.

En opinión de Cordero el Ordenamiento Territorial en Chile no ha sido desarrollado de acuerdo a una concepción adecuada, pero no niega su existencia, señalando que el Ordenamiento Territorial en nuestro país, tiene un alcance particular. Para Cordero el alcance del Ordenamiento

\footnotetext{
${ }^{8}$ CORREA, María Josefina, ver nota 1. op.cit. pág. 62

${ }^{9}$ CORDERO Quinzacara, Eduardo, "El Derecho urbanístico, los instrumentos de planificación territorial y el régimen jurídico de los bienes públicos”, Revista de Derecho de la Pontificia Universidad Católica de Valparaíso, XXIX, Valparaíso, Chile, 2007, [pp. 269-298] pág.294
} 
Sustainability, Agri, Food and Environmental Research 3(2), 2015: 12-21 ISSN: 0719-3726

Territorial es deficiente, sin embargo sí se ha tratado, pero más bien como una “... función pública o potestad específica que tenga por objeto una ordenación racional del espacio a nivel regional.

Sin embargo, en un sentido amplio constituye una función de los gobiernos regionales, para lo cual disponen de diversos Instrumentos de Planificación Territorial, como son los previstos en la Ley general de urbanismo y construcción ..."10, y sus falencias radican principalmente en que se ha anclado a una "visión urbana", que los niveles de planificación de las ciudades se han centrado principalmente en las comunas, y que las consideraciones ambientales a la luz de la evaluación ambiental (anterior a la vigencia de la Ley $N^{\circ}$ 20.417, que modificó la Ley General de Bases del Medio Ambiente $\mathrm{N}^{\circ}$ 19.300) se centraba principalmente en aspectos legalistas, de que los Instrumentos de Planificación Territorial no entraran en conflicto con las normas ambientales vigentes, en vez de considerar el aspecto holístico del medio ambiente y la condición de las personas como sus derechos constitucionales. ${ }^{11}$

Lo que en doctrina se plantea en síntesis es que en Chile se ha tendido a confundir al Ordenamiento Territorial con la Planificación Territorial, lo que a la luz de lo que hemos analizado es un error, ya que el primero encierra en su concepto una política que no solamente busca zonificar los usos del territorio y del suelo, sino que intenta fijar las directrices para una ordenación racional y equilibrada del territorio en base a distintos principios que consigan que las distintas actividades humanas se desarrollen en armonía en un territorio determinado. Sin perjuicio de lo que se ha considerado en la doctrina mayoritaria en Chile, de la falta de un concepto de Ordenamiento Territorial en nuestro país, no concordamos con ella, ya que de acuerdo a lo analizado a propósito del PROT, se ha avanzado en cuanto a la concepción del significado del mismo.

En efecto, el PROT recoge un concepto de Ordenamiento Territorial como "la expresión espacial de las políticas económicas, sociales, culturales y ecológicas de la sociedad. Es a la vez una disciplina científica, una técnica administrativa y una política concebida como un enfoque interdisciplinario y global, cuyo objetivo es un desarrollo equilibrado de las regiones y la organización física del espacio según un concepto rector" ${ }^{2}$, además ha establecido como principios de “descentralización, participación social, sustentabilidad urbana e integración social” ${ }^{13}$, desde la Carta Europea de Ordenación del Territorio, de 1983, con lo que busca conseguir la orientación de las

\footnotetext{
${ }^{10}$ Ver cita 107, CORDERO Quinzacara, Eduardo, op. cit. pág. 218.

${ }^{11}$ Ver cita 107, CORDERO Quinzacara, Eduardo, op. cit. pág. 219.

${ }^{12}$ PLAN REGIONAL DE ORDENAMIENTO TERRITORIAL: Contenido y Procedimientos, Subsecretaría de Desarrollo Regional y Administrativo, Departamento de Políticas y Descentralización, División Políticas y Estudios, abril 2011, Santiago de Chile, pág. 16.

${ }^{13}$ Op. cit. pág. 25
} 
Sustainability, Agri, Food and Environmental Research 3(2), 2015: 12-21

ISSN: 0719-3726

distintas actividades humanas para evitar el desequilibrio que deriva de la desarmonía que se desencadena por una superposición de éstas en un mismo espacio, que no cuentan con una adecuada planeación.

A pesar de contar con un Plan que no constituye ni una normativa ni una política, y que además se ocupa del territorio regional, serían factores a mejorar en camino del avance nacional hacia el Ordenamiento Territorial, elaborando una política nacional que comprendiera el territorio en su totalidad, para luego establecer el camino hacia los Planes Regionales, a través del PROT. Ello, recogiendo la idea de Cordero, en relación a desarrollar una política supralocal. ${ }^{14}$

\section{Planificación Territorial: concepto legal y doctrinario en Chile}

Concepto legal de la Planificación Territorial en Chile: La Constitución Política de la Republica, en su artículo $3^{\circ}$, se refiere a la administración del territorio en Chile, la cual será descentralizada o desconcentrada en su caso, radicando su administración en el Presidente de la República, según lo establece el artículo $24^{\circ}$ de la misma. En el caso de nuestra carta fundamental, no se habla de Planificación Territorial.

La Planificación Territorial se encuentra regulada en la Ley General de Urbanismo y Construcciones, LGUC, Decreto con Fuerza de Ley $\mathrm{N}^{\circ}$ 458, publicada en el Diario Oficial de 13 de abril de 1976, que en el título II, se refiere a "La Planificación Urbana", sin embargo, no se refiere a la Planificación Territorial propiamente tal.

La definición de Planificación Urbana contenida en la LGUC, la encontramos en el artículo $27^{\circ}$ de la misma, refiriendo que es “...el proceso que se efectúa para orientar y regular el desarrollo de los centros urbanos en función de una política nacional, regional y comunal de desarrollo socioeconómico". Se aprecia que esta definición deja de lado al espacio rural, el que sin embargo está tratado a propósito de la Planificación Urbana Intercomunal, referido expresamente al IPT Plan Regulador Intercomunal ${ }^{15}$. La LGUC en la cual los capítulos I y II hablan sobre la planificación urbana en particular.

\footnotetext{
${ }^{14}$ CORDERO Quinzacara, Eduardo, “El Derecho urbanístico, los instrumentos de planificación territorial el régimen jurídico de los bienes públicos”, op. cit. pág. 294

${ }^{15}$ Artículo $34^{\circ}$ LGUC. "Se entenderá por Planificación Urbana Intercomunal aquella que regula el desarrollo físico de las áreas urbanas y rurales de diversas comunas ..."
} 
Sustainability, Agri, Food and Environmental Research 3(2), 2015: 12-21 ISSN: 0719-3726

Por su parte, la Ordenanza General de Urbanismo y Construcciones (OGUC), Decreto $\mathrm{N}^{\circ} 47$, de 1992, del Ministerio de Vivienda y Urbanismo, no habla del concepto de Planificación Territorial, sino que más bien establece el reglamento de la LGUC en esta materia, con el objeto de regular la Planificación Urbana.

Por su parte la Política Nacional de Desarrollo Urbano, promulgada mediante Decreto $\mathrm{N}^{\circ} 78$, del Ministerio de Vivienda y Urbanismo, publicado en el Diario Oficial de 4 de marzo de 2014, tampoco define la Planificación Territorial, más bien hace referencia a la planificación urbana, relacionada al urbanismo como centro de atención de la política, asimilando a la tarea de los Órganos de la Administración del Estado hacia la gestión de la planificación urbana a través de los Instrumentos de Planificación Territorial, indicando que la "urbanística (que deriva de la palabra latina "urbs", "ciudad"), sino también desde un perspectiva más amplia, que tiende a la ordenación integral del territorio, prisma bajo el cual se distingue como la ciencia de la ordenación de las ciudades y del territorio, siendo su objeto principal la planificación de las mismas (esto es "la planeación urbana", término introducido por Hipodamo de Mileto, considerado el padre del urbanismo), los estudios sobre el fenómeno urbano, la acción de urbanización y la organización de la ciudad y del territorio." 16 de lo que podemos inferir que esta política nacional se refiere también a la ordenación del territorio, abocada a la planificación de ciudades y del territorio, entendiendo su influencia en el espacio global que comprende lo nacional, regional, provincial y comunal.

Sin embargo, tampoco trata expresamente el Ordenamiento Territorial, existiendo solamente esta referencia en su texto. En síntesis, y siempre considerando que se acerca más a una concepción de Ordenamiento Territorial, ésta señala que es necesario contar con una política que fije las directrices y marco estructural de las materias de urbanismo, desarrollo territorial, vivienda y edificación pública, al señalar que "tales avances podrían ser mayores y más efectivos de contar nuestra institucionalidad- con un marco explícito de principios, objetivos y lineamientos contenidos en una Política Nacional de Desarrollo Urbano, que sirva de orientación e integración de las iniciativas en materia de urbanismo, desarrollo territorial - obras urbanas, vivienda, y edificación pública." 17

Como podemos apreciar, nuestro ordenamiento jurídico no define un concepto de Planificación Territorial propiamente tal, careciendo de una conceptualización precisa. No obstante,

\footnotetext{
${ }^{16}$ DECRETO N ${ }^{\circ} 78$, Ministerio de Vivienda y Urbanismo, publicado en Diario Oficial de 4 de marzo de 2014. Capítulo I En Materia de Urbanismo.

${ }^{17}$ Op. cit. Considerando a).
} 
Sustainability, Agri, Food and Environmental Research 3(2), 2015: 12-21 ISSN: 0719-3726

sí considera la gestión de Planificación Territorial, confundiéndolo con la planificación urbana, regulando los tipos de Instrumentos de Planificación Territorial que aplican en los distintos niveles o escalas territoriales descritos por el artículo 28 de la LGUC, nacional, regional, intercomunal y comunal.

Concepto doctrinario de Planificación Territorial en Chile: La doctrina mayoritariamente se inclina por señalar que la conceptualización sobre Planificación Territorial no es homogénea ni se encuentra específicamente definida, ya que no existe una definición concreta en el ordenamiento jurídico, ya que es confundida con el urbanismo, pues está manifiestamente claro que la Ley de Urbanismo y Construcciones no se refiere a la planificación en general, sino solamente a la urbana, a pesar que sí regula territorios rurales a través de los PRI (Planes Reguladores Intercomunales).

Cordero $^{18}$ explica que la planificación nace desde la necesidad de incorporar una pluralidad de factores en las cuales indique la regulación del espacio, que alcanza diferentes ámbitos como el medio ambiental, económico, social, cultural, los cuales sobrepasan un ámbito local, por lo que se requiere de una planificación del territorio, que a nivel superior pueda analizar los diferentes ámbitos de incidencia sobre éste.

Asimismo, Cordero señala que la planificación es un instrumento necesario para incorporar la idea de utilización racional del territorio, como una planificación de los espacios. En particular dice que "en Chile la Planificación Territorial en todos sus niveles y contenidos, va a estar necesariamente condicionada por el marco constitucional y el orden de valores que este consagra, al cual debe responder la acción de la Administración del Estado: contribuir a crear las condiciones sociales que permitan la mayor realización espiritual y material posible de todos los habitantes de la nación..."19 en particular la observancia del principio básico que consiste en la búsqueda de un desarrollo territorial armónico y equitativo, como dispone el artículo $115^{\circ}$ de la Constitución Política.

En este sentido, indica que existen dificultades en torno a lo que establece la Constitución y nuestro ordenamiento jurídico en la materia, ya que por una parte la LGUC está centrada en una visión eminentemente urbana, ya que esta considera los distintos niveles de planificación, nacional, regional, intercomunal y comunal, enfocando su objetivo principal en los procesos de urbanización y edificación; a su vez, en relación los niveles de planificación de ciudades en Chile fundamentalmente

\footnotetext{
${ }^{18}$ CORDERO Quinzacara, Eduardo, "Ordenamiento territorial, justicia ambiental y zonas costeras" Revista de Derecho de la Pontificia Universidad Católica de Valparaíso XXXVI (Valparaíso, Chile, 2011, 1er Semestre) [pp. 209 - 249]

${ }^{19}$ Op. cit. pág. 219.
} 
Sustainability, Agri, Food and Environmental Research 3(2), 2015: 12-21 ISSN: 0719-3726

se ha desarrollado en el nivel comunal, existiendo pocos instrumentos a nivel intercomunal; y por último la Planificación Territorial aun no es capaz de incorporar adecuadamente las consideraciones ambientales, a pesar de la existencia del instrumento de gestión ambiental de la Evaluación Ambiental Estratégica, incorporado en la Ley $\mathrm{N}^{\circ} 19.300$ sobre bases generales del Medio Ambiente, en su artículo 7 bis.

Para Andrade, Arenas y Guijón ${ }^{20}$, la Planificación Territorial es el proceso de desarrollo de planes y medidas descritos por un modelo territorial futuro que idealmente se encontrará en una política de Ordenamiento Territorial, que defina objetivos y directrices del desarrollo territorial de un espacio determinado, concluyendo que las normas jurídicas que se dictan teniendo como objetivos el ordenamiento o la Planificación Territorial serían las normas de Ordenamiento Territorial o los Instrumentos de Planificación Territorial. En consecuencia, la Planificación Territorial, quedaría en un nivel de regulación al igual que el Ordenamiento Territorial.

Gastó por su parte, piensa que la planificación es un proceso para establecer un marco regulador general para lograr las condiciones óptimas en relación a la calidad de vida de la población, señalando que un "Plan" posee "una visión holística del Ordenamiento Territorial, con una perspectiva largoplacista." ${ }^{21}$, de esta manera le otorga una concepción similar a la que se podría concebir como Ordenamiento Territorial, el cual posee las características de establecer un marco directriz que fija los objetivos y principios sobre el desarrollo del territorio. Sin embargo, se refiere a la Planificación Territorial un carácter regulador, pues tal vez se está refiriendo a los IPT propiamente tales. En Barton encontramos otro concepto de Planificación Territorial, entendida como "La Planificación Territorial de ciudades-regiones es un ejercicio de anticipación para reducir riesgos y, a la vez, promover bienestar; no es solamente un proceso para minimizar la vulnerabilidad, sino también para identificar formas de maximizar oportunidades" 22.

Considerando las diferentes opiniones de la doctrina en relación a la Planificación Territorial en Chile, podemos concluir que es la materialización de las políticas de desarrollo territorial en un espacio determinado, como regional, intercomunal y local, con carácter generalmente regulador o normativo.

\footnotetext{
${ }^{20}$ ANDRADE Belisario, ARENAS Federico, GUIJON Rodrigo, "Revisión crítica del marco institucional y legal chileno de ordenamiento territorial, el caso de la zona costera", op. cit. pág. 25

${ }^{21}$ GASTÓ Juan, RODRIGO Patricia, ARANGUIZ Ivonne, URRUTIA Carola, op. cit. pág. 38

${ }^{22}$ LIRA, L. "Revalorización de la planificación del desarrollo". Santiago: CEPAL, 2006, en BARTON, Jonathan, "Adaptación al cambio climático en la planificación de ciudades-regiones", Revista de Geografía Norte Grande, 43: 5-30, 2009.
} 
Sustainability, Agri, Food and Environmental Research 3(2), 2015: 12-21 ISSN: 0719-3726

En síntesis, la Planificación Territorial es el proceso de regulación de los usos de un territorio definido en sus diferentes niveles (regional, intercomunal y local), para la consecución de los objetivos delineados por el Ordenamiento Territorial en el espacio físico, lo que es en definitiva, la concreción de los fines descritos por una política de Ordenamiento Territorial. Esta Planificación Territorial, como señala Cordero, incorporará las diferentes materias que incidirán en el territorio, como por ejemplo las zonas de protección, zonas de riesgo, los usos del suelo, el diseño vial, etc.

\section{Diferencias entre el Ordenamiento Territorial y la Planificación Territorial en Chile,} de acuerdo a las definiciones doctrinarias y legales.

La principal diferencia entre el Ordenamiento Territorial y la Planificación Territorial, es que mientras el primero goza de características indicativas, directrices, que definirán los objetivos y principios que se regularán a futuro un espacio determinado, la segunda está supeditada al Ordenamiento Territorial, cuyo fin es implementar y ejecutar en una escala o nivel territorial determinado, las directrices fijadas por el mismo, que podrá estar contenido en una política o una normativa. Sin embargo en Chile, no existe normativa o fuente formal, pues como ya analizamos, se está trabajando en un Plan Regional de Ordenamiento Territorial -PROT, función que detentan los Gobiernos Regionales y la SUBDERE.

Además podemos colegir que entre el Ordenamiento y Planificación Territorial existe una relación de género a especie, en donde el Ordenamiento Territorial es el género y la Planificación Territorial es la especie. Si bien existen diferencias, desde sus funciones específicas, están relacionados mutuamente. El primero tiene por función ser la expresión de la potestad del Estado de administrar el territorio de la nación, el segundo es la materialización en el espacio de las políticas pre establecidas para la regulación del territorio a escala regional, intercomunal y local, como ya describimos.

Por último, reconocemos que con los avances conseguidos en nuestro país, con la elaboración del PROT, se han establecido algunas directrices y objetivos para el desarrollo armónico y equitativo de las regiones del país, definiendo los principios fundamentales y lineamientos que deberán incorporarse en los futuros instrumentos de Ordenamiento Territorial. En este sentido, actualmente nuestro país cuenta con un instrumento de Ordenamiento Territorial Regional, PROT, que sin embargo no ha sido reflejado en una normativa que pueda regular un territorio determinado, como tampoco se ha traducido en una política de carácter de vinculante. 